August, 1993

ITP-SB-93-44

RI-152-93

hep-th/9308154

\title{
On Nonabelian Duality
}

\author{
Amit Giveon日 \\ Racah Institute of Physics, The Hebrew University \\ Jerusalem, 91904, ISRAEL \\ and \\ Martin Roček P \\ Institute for Theoretical Physics \\ State University of New York at Stony Brook \\ Stony Brook, NY 11794-3840 USA
}

\begin{abstract}
We show that nonabelian duality is not a symmetry of a conformal field theory, but rather a symmetry between different theories. We expose a nonlocal symmetry of nonabelian dual theories. We show how, in the case with vanishing isotropy, it can be used to find the inverse dual transformation. Finally, we consider a number of new examples.
\end{abstract}

\footnotetext{
${ }^{1}$ e-mail address: giveon@vms.huji.ac.il

${ }^{2}$ e-mail address: rocek@insti.physics.sunysb.edu
} 


\section{Introduction}

A great deal of insight into the structure of the moduli space of string backgrounds and conformal field theories (CFT's) has been uncovered by studies of target space duality (see, for example [1], 2, 3]). The $O(d, d, \mathbf{Z})$ symmetry of the CFT's defined on backgrounds with $d$ toroidal isometries can be realized by duality transformations of the type studied by T. Buscher [ [⿴囗十 . These are found by gauging a symmetry of the action with nondynamical gauge fields, i.e., without a $F^{2}$ term, and adding a Lagrange multiplier that constrains the gauge field to be (locally) pure gauge. After being careful with the global issues, such dualities can be made into exact symmetries of the CFT [5].

In his thesis, T. Buscher [6] also considered the possibility of performing duality transformations for nonabelian symmetries. [3 More recently, de la Ossa and Quevedo [9] have independently proposed the same idea, and explored a class of $S O(3)$ examples. However, they did not consider exact conformal backgrounds (e.g., WZNW models), and did not analyze the global issues.

Here we consider nonabelian duality for CFT's. We argue that, except for "accidents", there is no reason to expect nonabelian duality to be a symmetry of a CFT; at best, it can be a transformation between different CFT's. We discover a nonlocal symmetry of dual theories. We discuss the crucial role of isotropy, and consider several explicit examples for exact CFT's.

The structure of the paper is as follows: In section $\mathbf{2}$, we begin with a review of Abelian duality. In section 3, we consider general aspects of nonabelian duality, and present our argument about the problem with global issues. Whereas in the Abelian case, the dual theory has a natural symmetry suitable for inverting the duality transformation, in the nonabelian case we find that this symmetry becomes nonlocal and cannot obviously be used for the inverse. In section $\mathbf{4}$, we specialize to the case without isotropy, and in section $\mathbf{5}$, we give a few examples. In section $\mathbf{6}$, we explicitly work out the

\footnotetext{
${ }^{3}$ Earlier examples have been discussed by Fridling and Jevicki [7] and by Fradkin and Tseytlin [8].
} 
$S U(2)$ dual of the $S U(2)-W Z N W$ model (a case with isotropy). We close with some conclusions and a brief discussion.

\section{Review of Abelian Duality}

In this section, we briefly review Abelian duality [由, 5]. We begin with a target space geometry with a compact Abelian symmetry. The duality transformation is performed by gauging this symmetry and adding a Lagrange multiplier term that constrains the gauge field to be pure gauge. Integrating out the lagrange multiplier gives back the original model, and integrating out the gauge field gives the dual model.

Without loss of generality, we can choose coordinates on the target space where the symmetry acts by shifts of a single periodic coordinate $\theta \equiv \theta+2 \pi$, and the remaining coordinates $x^{i}$ are left inert. In these coordinates, the background is independent of $\theta$. The action of the original model takes the form

$$
\begin{aligned}
S[\theta, x]=\frac{1}{2 \pi} \int d^{2} z(E(x) \partial \theta \bar{\partial} \theta & +F_{j}^{R}(x) \partial \theta \bar{\partial} x^{j}+F_{i}^{L}(x) \partial x^{i} \bar{\partial} \theta \\
& \left.+F_{i j}(x) \partial x^{i} \bar{\partial} x^{j}-\frac{1}{4} \Phi(x) R^{(2)}\right)
\end{aligned}
$$

where $\Phi$ is the dilaton field and $R^{(2)}$ is the world sheet curvature. We now gauge the symmetry by minimal coupling $\partial \theta \rightarrow \partial \theta+A, \bar{\partial} \theta \rightarrow \bar{\partial} \theta+\bar{A}$, and add a Lagrange multiplier term

$$
\frac{1}{2 \pi} \int d^{2} z(A \bar{\partial} \lambda-\bar{A} \partial \lambda)
$$

up to an important total derivative, this is just $\lambda F, F(A, \bar{A}) \equiv \partial \bar{A}-\bar{\partial} A$. When $\lambda$ is chosen to have periodicity $2 \pi$, as discussed in [5], the winding modes of $\lambda$ constrain the holonomy of $A, \bar{A}$ to insure that integrating out $\lambda$ gives back the original model.t.

\footnotetext{
${ }^{4}$ In [5], though the discussion of the winding modes of $\lambda$ is correct, the Lagrange multiplier term was written as $\lambda F$, and thus the total derivative term needed to make the winding modes contribute was accidentally omitted.
} 
In this form, it is clear that the dual model has a $U(1)$ symmetry that acts on $\lambda$ by

$$
\lambda \rightarrow \lambda+\epsilon,
$$

for $\epsilon$ constant. If we make a duality transformation with respect to this symmetry, we immediately see that the dual of the dual is the original model: We gauge (2.3) and add a second Lagrange multiplier $\tilde{\lambda}$ :

$$
\begin{aligned}
S[x, A, \lambda] & \rightarrow S[x, A, \tilde{A}, \lambda, \tilde{\lambda}] \\
& \equiv S[x, A, \lambda]+\frac{1}{2 \pi} \int d^{2} z \operatorname{tr}(\tilde{\bar{A}} A-\tilde{A} \bar{A}+\tilde{A} \bar{\partial} \tilde{\lambda}-\tilde{\bar{A}} \partial \tilde{\lambda}) .
\end{aligned}
$$

Integrating out $\tilde{A}, \tilde{\bar{A}}$ gives:

$$
A=\partial \tilde{\lambda}, \quad \bar{A}=\bar{\partial} \tilde{\lambda},
$$

and hence we recover the original model. The inverse transformation is a sign of an underlying group of duality transformations. For $d$ commuting $U(1)$ symmetries, one finds an $O(d, d, \mathbf{Z})$ group [3].

For the case of one symmetry, choosing a gauge $\theta=0$, we find the gauged action

$$
\begin{aligned}
S[x, A, \lambda]=\frac{1}{2 \pi} \int d^{2} z(E A \bar{A} & +F_{i}^{R} A \bar{\partial} x^{i}+F_{i}^{L} \partial x^{i} \bar{A}+F_{i j} \partial x^{i} \bar{\partial} x^{j} \\
& \left.-\frac{1}{4} \Phi R^{(2)}+(A \bar{\partial} \lambda-\bar{A} \partial \lambda)\right) .
\end{aligned}
$$

Integrating out the gauge field $A, \bar{A}$, we find

$$
A(\lambda, x)=\left(\partial \lambda-\partial x^{i} F_{i}^{L}\right) E^{-1}, \quad \bar{A}(\lambda, x)=-E^{-1}\left(\bar{\partial} \lambda+F_{i}^{R} \bar{\partial} x^{i}\right) .
$$

Substituting (2.7) into the gauged action (2.6), we find the dual action:

$$
\begin{array}{r}
\tilde{S}[x, \lambda]=\frac{1}{2 \pi} \int d^{2} z \\
\left(\left(\partial \lambda-\partial x^{i} F_{i}^{L}\right) E^{-1}\left(\bar{\partial} \lambda+F_{i}^{R} \bar{\partial} x^{i}\right)\right. \\
\left.+F_{i j} \partial x^{i} \bar{\partial} x^{j}-\frac{1}{4}(\Phi+\ln E) R^{(2)}\right) .
\end{array}
$$

The shift of the dilaton comes from a Jacobian factor that arises from integrating over the gauge field $A, \bar{A}$ [4]. 
A general feature of duality is that field equations and Bianchi identities are rotated into each other [10]. Here, they are simply interchanged.

In the original model (2.1), the field equation and Bianchi identity are:

$$
\text { Field Equation : } \quad \bar{\partial} J+\partial \bar{J}=0
$$

for

$$
J=E \partial \theta+F_{i}^{L} \partial x^{i}, \quad \bar{J}=E \bar{\partial} \theta+F_{i}^{R} \bar{\partial} x^{i},
$$

and

Bianchi Identity : $\quad \partial\left[E^{-1}\left(\bar{J}-F_{i}^{R} \bar{\partial} x^{i}\right)\right]-\bar{\partial}\left[\left(J-\partial x^{i} F_{i}^{L}\right) E^{-1}\right]=0$

(substituting the definition of $J, \bar{J}$ (2.10) into (2.11), this becomes just the triviality $\partial \bar{\partial} \theta-\bar{\partial} \partial \theta=0)$.

In the dual model, we have the obvious currents

$$
\tilde{J}=\partial \lambda, \quad \tilde{\bar{J}}=-\bar{\partial} \lambda .
$$

These obey the dual Bianchi identity:

$$
\text { Dual Bianchi Identity : } \quad \bar{\partial} \tilde{J}+\partial \tilde{\bar{J}}=0 .
$$

By construction, the $\lambda$ field equation is $F(A, \bar{A})=0$, with $A, \bar{A}$ given in (2.7); in terms of the dual currents $\tilde{J}, \tilde{\bar{J}}$, this takes the form:

Dual Field Equation : $\quad \partial\left[E^{-1}\left(\tilde{\bar{J}}-F_{i}^{R} \bar{\partial} x^{i}\right)\right]-\bar{\partial}\left[\left(\tilde{J}-\partial x^{i} F_{i}^{L}\right) E^{-1}\right]=0$.

Thus we see that the field equation (2.9) becomes the dual Bianchi identity (2.13) and the Bianchi identity (2.11) becomes the dual field equation (2.14).

\section{General Aspects of Nonabelian Duality}

As in the Abelian case, we begin by gauging some (non-anomalous) symmetry of the target space geometry, and then add a Lagrange multiplier term that constrains the field strength of the gauge field to vanish [6], [9]:

$$
S\left[x^{a}\right] \rightarrow S[x, A, \lambda] \equiv S\left[x^{a}, A, \bar{A}\right]+\frac{1}{2 \pi} \int d^{2} z \operatorname{tr}(\lambda F(A, \bar{A})) .
$$


Here

$$
F(A, \bar{A}) \equiv \partial \bar{A}-\bar{\partial} A+[A, \bar{A}]
$$

is the field strength for the world-sheet gauge field $(A, \bar{A})$ and $x^{a}$ are the scalar fields of the original action. We now consider how much of our discussion of the Abelian case carries over.

As in the Abelian case, when we integrate out $\lambda$, we find that locally, $A=h^{-1} d h$ is pure gauge, and hence, modulo global issues, we recover the original model.

We next consider the question of the holonomy. Here, there are three immediate and crucial differences. (1) Whereas in the Abelian case $\lambda$ is a gauge singlet that can naturally be chosen to be periodic, and thus acquire the winding modes that act as the Lagrange multipliers for the holonomies of $A$, in the nonabelian case, $\lambda$ transforms in the adjoint representation, and in general the group action is incompatible with making $\lambda$ a periodic variable. (2) Even if one found some way to introduce winding modes for $\lambda$, they would not multiply the holonomies of $A$, since in the nonabelian case, these are path-ordered exponentials, and hence cannot be written in a local form as terms in the action. (3) Finally, the $\operatorname{tr} \lambda[A, \bar{A}]$ term is incompatible with trying to write the action in a form analogous to (2.2). Consequently, we conclude that nonabelian duality is not an invariance of the underlying conformal field theory, but rather we conjecture that it is a transformation between different conformal field theories related by a (infinite order) nonabelian orbifold construction.

In the Abelian case, the invariance of the action under constant shifts of

\footnotetext{
${ }^{5}$ In the Abelian case, if one neglects to compactify $\lambda$, when one integrates it out, one gets $A=d \theta$ for noncompact $\theta$; modding out by the (infinite order) group of translations of $\theta$ by $2 \pi$, one recovers the original model. Equivalently, modding out the original model by the continuous group $U(1)$ gives rise to continuous twisted sectors that decompactify $\theta$. Similarly, in the nonabelian case, since we cannot compactify $\lambda$, we would need to twist the model that we get by integrating out $\lambda$ (the "dual CFT") by some infinite order group analogous to the shifts of $\theta$ to recover the original model. Equivalently, we can think of the dual CFT as the $G$ orbifold of the original CFT, where $G$ is the continuous group of symmetries that we used for nonabelian duality.
} 
$\lambda$ is crucial: it guarantees that the dual geometry has a symmetry that can be used for a further duality transformation, and that the dual of the dual is the original model (see 2.4, 2.5). In the nonabelian case, this symmetry becomes nonlocal, and no longer serves either function: the dual geometry need not have a local symmetry, and if one attempts to dualize the nonlocal symmetry, one does not appear to recover the original model.

To see the nonlocal symmetry, we introduce nonlocal variables to describe the gauge field:

$$
\begin{aligned}
& h(\zeta)=h_{0} P \exp \left(\int^{\zeta} A d z\right) \quad \Rightarrow \quad A=h^{-1} \partial h, \\
& \bar{h}(\bar{\zeta})=\bar{h}_{0} P \exp \left(\int^{\bar{\zeta}} \bar{A} d \bar{z}\right) \quad \Rightarrow \quad \bar{A}=\bar{h}^{-1} \bar{\partial} \bar{h},
\end{aligned}
$$

where $P$ denotes path ordering. Then the field strength $F$ can be rewritten as:

$$
F(A, \bar{A})=h^{-1} \partial\left(f^{-1} \bar{\partial} f\right) h=\bar{h}^{-1} \bar{\partial}\left(\partial f f^{-1}\right) \bar{h}, \quad f \equiv \bar{h} h^{-1} .
$$

This implies that

$$
\operatorname{tr} \lambda F=\operatorname{tr}\left(h \lambda h^{-1}\right) \partial\left(f^{-1} \bar{\partial} f\right)=\operatorname{tr}\left(\bar{h} \lambda \bar{h}^{-1}\right) \bar{\partial}\left(\partial f f^{-1}\right),
$$

and hence the action (3.1) is invariant under the nonlocal Kač-Moody transformations

$$
\delta \lambda=h^{-1} \bar{\epsilon}(\bar{z}) h+\bar{h}^{-1} \epsilon(z) \bar{h} .
$$

These are highly nonlocal transformations in terms of the original fields $A, \bar{A}$ (see 3.3 ), and hence, after $A, \bar{A}$ are eliminated by their algebraic equations of motion, these are nonlocal in terms of the coordinates of the dual geometry.

In the Abelian case, we were able to immediately see that the dual of the dual is the original model by gauging the translation symmetry of $\lambda$ and adding a $\tilde{\lambda} F(\tilde{A}, \tilde{\bar{A}})$ term (see 2.4). We can attempt to do the same thing here by gauging the nonlocal shift symmetry of the action $S[x, A, \lambda]$ (3.1):

$$
\begin{aligned}
S[x, A, \lambda] & \rightarrow S[x, A, \tilde{A}, \lambda, \tilde{\lambda}] \\
& \equiv S[x, A, \lambda]+\frac{1}{2 \pi} \int d^{2} z \operatorname{tr}\left(\tilde{A} f^{-1} \bar{\partial} f+\tilde{\bar{A}} \partial f f^{-1}+\tilde{\lambda}(\partial \tilde{\bar{A}}-\bar{\partial} \tilde{A})\right) .
\end{aligned}
$$


This action is invariant under the nonlocal gauge transformations

$$
\delta \tilde{A}=\partial \epsilon(z, \bar{z}), \quad \delta \tilde{\bar{A}}=\bar{\partial} \epsilon(z, \bar{z}), \quad \delta \lambda=h^{-1} \epsilon(z, \bar{z}) h+\bar{h}^{-1} \epsilon(z, \bar{z}) \bar{h}
$$

Integrating out $\tilde{\lambda}$ restricts $\tilde{A}, \tilde{\bar{A}}$ to be pure gauge (at least locally), and gives back $S[x, A, \lambda]$ after an obvious nonlocal redefinition of $\lambda$. Unfortunately, integrating out $\tilde{A}, \tilde{\bar{A}}$ does not yield the original model; in particular, we do not find $F(A, \bar{A})=0$ is necessary, and we get a model from which we cannot eliminate the various auxiliary degrees of freedom that we have introduced along the way.

There is of course a simple way to add terms analogous to the Abelian case (2.4) to the action $S[x, A, \lambda]$ (3.1) so that integrating out $\tilde{A}, \tilde{\bar{A}}$ gives back the original model:

$$
\begin{aligned}
S[x, A, \lambda] & \rightarrow S[x, A, \tilde{A}, \lambda, \gamma] \\
& \equiv S[x, A, \lambda]+\frac{1}{2 \pi} \int d^{2} z \operatorname{tr}\left(\tilde{\bar{A}}\left(A-\gamma^{-1} \partial \gamma\right)-\tilde{A}\left(\bar{A}-\gamma^{-1} \bar{\partial} \gamma\right)\right)
\end{aligned}
$$

Unfortunately, integrating out $\gamma$ does not allow us to eliminate $\tilde{A}, \tilde{\bar{A}}$; furthermore, after integrating out $A, \bar{A}$ and fixing the gauge invariance, in general we do not know how to look at the resulting model and introduce $\tilde{A}, \tilde{\bar{A}}$ and $\gamma$.

There is another issue that does not arise in the Abelian case, but is crucial here: nonabelian gauge groups may have nontrivial isotropy subgroups, that is, they may act on the target space coordinates $x^{a}$ so that one cannot completely fix the gauge by conditions on $x^{a}$. (For example, this is the case for the vector action of a group $G$ on the $G$-WZNW model; for another class of examples, see [9].) In this case, in the dual model, some of the dual coordinates $\lambda$ must be gauge fixed, and a clear separation into original and dual coordinates is impossible. 


\section{The case without isotropy}

When the isotropy vanishes, i.e., when the gauge symmetry can be fixed completely by conditions on the coordinates of the original model, some more aspects of nonabelian duality resemble the Abelian case. This is the generic situation for WZNW models: For a group $G$ dualized with respect to a proper subgroup $H$, e.g. $G=S U(N), H=S U(N-m), m \neq 0$, the isotropy vanishes at generic points of $G$; on certain singular subspaces, it becomes nonvanishing; at these points, the dual space has singularities, which, however, are not peculiar from the viewpoint of the underlying CFT.

\subsection{The original model.}

In general, we can consider a target space with coordinates $g$ that transform as $g \rightarrow u^{-1} g$ for $u$ in some group $G$, and further coordinates $x^{i}$ that are inert.f A general non-anomalous action can be written in the form

$$
\begin{aligned}
S[g, x]=\frac{1}{2 \pi} \int d^{2} z\left(E_{a b}(x)\left(g^{-1} \partial g\right)^{a}\left(g^{-1} \bar{\partial} g\right)^{b}\right. & +F_{a j}^{R}(x)\left(g^{-1} \partial g\right)^{a} \bar{\partial} x^{j} \\
+F_{i b}^{L}(x) \partial x^{i}\left(g^{-1} \bar{\partial} g\right)^{b} & +F_{i j}(x) \partial x^{i} \bar{\partial} x^{j} \\
& \left.-\frac{1}{4} \Phi(x) R^{(2)}\right)
\end{aligned}
$$

where

$$
\left(g^{-1} \partial g\right)^{a} \equiv \operatorname{tr}\left(T^{a} g^{-1} \partial g\right) \Leftrightarrow g^{-1} \partial g=\left(g^{-1} \partial g\right)^{a} T_{a}, \quad \text { etc. },
$$

and the generators $T_{a}, a=1, \ldots, \operatorname{dim}(G)$, obey

$$
\operatorname{tr}\left(T_{a} T_{b}\right)=\eta_{a b}
$$

\footnotetext{
${ }^{6}$ Any geometry with vanishing isotropy can be parametrized by coordinates $g$ and $x^{i}$ because when the isotropy vanishes, we can fix the gauge completely by algebraic conditions on the coordinates of the target space. We call the remaining coordinates, whatever they are, $x^{i}$. We then transform to a general gauge with a parameter $g$ which becomes our coordinate $g$. By the group property, subsequent (passive) gauge transformations then transform $g$ correctly and leave $x^{i}$ inert. For the example $G / H$ mentioned above, we would write an element $g_{G} \in G$ in terms of $g \equiv g_{H} \in H$ and $x$ as $g_{G}=g_{H} x g_{H}^{-1}$.
} 
for some invariant metric $\eta$ that can be used to raise and lower indices. Under an infinitesimal variation $\delta g=\epsilon^{a} T_{a} g$, we find the field equation

$$
\partial \bar{J}+\bar{\partial} J=0
$$

where the currents $J, \bar{J}$ are

$$
\begin{aligned}
J=g \mathcal{J} g^{-1}, & \bar{J}=g \overline{\mathcal{J}} g^{-1}, \\
\mathcal{J}^{a}=\left(g^{-1} \partial g\right)^{b} E_{b}{ }^{a}+\partial x^{i} F_{i}^{L a}, & \overline{\mathcal{J}}^{a}=E^{a}{ }_{b}\left(g^{-1} \bar{\partial} g\right)^{b}+F_{i}^{R a} \bar{\partial} x^{i} .
\end{aligned}
$$

The currents $J, \bar{J}$ obey Bianchi identities which follow from

$$
F\left(g^{-1} \partial g, g^{-1} \bar{\partial} g\right)=0
$$

where $F$ is the field strength (3.2). In terms of $J, \bar{J}$, this involves explicit factors of $g$, and hence the form is explicitly gauge dependent; this can be cured by rewriting the identities in terms of the conjugated currents $\mathcal{J}, \overline{\mathcal{J}}$. They become just

$$
\text { Bianchi Identity : } \quad F(V(g, x), \bar{V}(g, x))=0,
$$

$$
V^{a}(g, x)=\left(\mathcal{J}^{b}-\partial x^{i} F_{i}^{L b}\right) E_{b}^{-1 a}, \quad \bar{V}^{a}(g, x)=E_{b}^{-1 a}\left(\overline{\mathcal{J}}^{b}-F_{i}^{R b} \bar{\partial} x^{i}\right) .
$$

Now the field equations (4.4) become:

Field Equation : $\quad \partial \overline{\mathcal{J}}+\bar{\partial} \mathcal{J}+[V, \overline{\mathcal{J}}]+[\bar{V}, \mathcal{J}]=0$.

\subsection{The dual model.}

To find the dual, we gauge the action $S[g, x]$ (4.1) by minimal coupling:

$$
g^{-1} \partial g \rightarrow g^{-1}(\partial+A) g, \quad g^{-1} \bar{\partial} g \rightarrow g^{-1}(\bar{\partial}+\bar{A}) g .
$$

Here $A$ transforms as $A \rightarrow u^{-1}(\partial+A) u$, etc. Adding the $\lambda F$ term, and choosing the gauge $g=1$, we get the action

$$
\begin{aligned}
S[x, A, \lambda]=\frac{1}{2 \pi} \int d^{2} z\left(E_{a b} A^{a} \bar{A}^{b}\right. & +F_{a i}^{R} A^{a} \bar{\partial} x^{i}+F_{i a}^{L} \partial x^{i} \bar{A}^{a}+F_{i j} \partial x^{i} \bar{\partial} x^{j} \\
& \left.-\frac{1}{4} \Phi R^{(2)}+\lambda_{a}\left(\partial \bar{A}^{a}-\bar{\partial} A^{a}+f_{b c}^{a} A^{b} \bar{A}^{c}\right)\right)
\end{aligned}
$$


Integrating out the gauge fields $A, \bar{A}$, we get

$$
\begin{aligned}
& A^{a}(\lambda, x)=\left(\partial \lambda_{b}-\partial x^{i} F_{i b}^{L}\right)\left[\left(E+\lambda_{c} f^{c}\right)^{-1}\right]^{b a}, \\
& \bar{A}^{a}(\lambda, x)=-\left[\left(E+\lambda_{c} f^{c}\right)^{-1}\right]^{a b}\left(\bar{\partial} \lambda_{b}+F_{b i}^{R} \bar{\partial} x^{i}\right),
\end{aligned}
$$

where the matrices $f^{c}$ have the structure constants as components $\left(f^{c}\right)_{a b}=$ $f_{a b}^{c}$. We find the dual theory

$$
\begin{array}{r}
\tilde{S}[x, \lambda]=\frac{1}{2 \pi} \int d^{2} z\left(\left(\partial \lambda_{b}-\partial x^{i} F_{i b}^{L}\right)\left[\left(E+\lambda_{c} f^{c}\right)^{-1}\right]^{b a}\left(\bar{\partial} \lambda_{a}+F_{a j}^{R} \bar{\partial} x^{j}\right)\right. \\
\left.+F_{i j} \partial x^{i} \bar{\partial} x^{j}-\frac{1}{4}\left[\Phi+\ln \left(\operatorname{det}\left[E+\lambda_{c} f^{c}\right]\right)\right] R^{(2)}\right)
\end{array}
$$

where the transformation of the dilaton comes from the Jacobian as in (2.8).

In the Abelian case, duality interchanges the field equations and the Bianchi identities (2.11); it is instructive to see what happens here.

The dual field equations (from the variation of $\lambda$ ) are precisely

$$
\text { Dual Field Equation : } \quad F(A(\lambda, x), \bar{A}(\lambda, x))=0
$$

for $A, \bar{A}$ given by (4.11); this has exactly the same form as the Bianchi identity (4.7) in the original model, and suggests that we identify "currents" $\tilde{\mathcal{J}}, \tilde{\mathcal{J}}$ :

$$
\tilde{\mathcal{J}}^{a}(\lambda, x)=A^{b} E_{b}{ }^{a}+\partial x^{i} F_{i}^{L a}, \quad \tilde{\overline{\mathcal{J}}}^{a}(\lambda, x)=E^{a}{ }_{b} \bar{A}^{b}+F_{i}^{R a} \bar{\partial} x^{i},
$$

with $A, \bar{A}$ given by (4.11). The dual "Bianchi" identity is the triviality $\partial \bar{\partial} \lambda=$ $\bar{\partial} \partial \lambda$; however, when this is written in terms of $\tilde{\mathcal{J}}, \tilde{\mathcal{J}}$, it becomes nontrivial. Using (4.11,4.14), we write $\partial \lambda, \bar{\partial} \lambda$ as

$$
\partial \lambda=\tilde{\mathcal{J}}+[\lambda, A], \quad \bar{\partial} \lambda=-\tilde{\overline{\mathcal{J}}}+[\lambda, \bar{A}],
$$

and then find

Dual Bianchi Identity : $\quad \partial \tilde{\overline{\mathcal{J}}}+\bar{\partial} \tilde{\mathcal{J}}+[A, \tilde{\mathcal{J}}]+[\bar{A}, \tilde{\mathcal{J}}]=[\lambda, F(A, \bar{A})]$

The right hand side is proportional to the dual field equation (4.13); the remaining term has the form of the field equation (4.8) in the original model. 


\subsection{Some comments on the duality group.}

In the Abelian case, when there is more than one isometry, one finds a duality group [1, 3]. Some of the generators of this group act by adding a total derivative to the Lagrangian; this shifts the antisymmetric tensor (KalbRamond field). Here we will find certain shifts of a similar type that lead to dual backgrounds that differ merely by coordinate transformations (shifts of $\lambda)$. Indeed, the dual action $\tilde{S}[x, \lambda]$ (4.12) has the following symmetry:

$$
\begin{gathered}
\lambda \rightarrow \lambda-\xi(x), \\
E_{a b} \rightarrow E_{a b}+\xi_{c} f_{a b}^{c}, \quad F_{a i}^{R} \rightarrow F_{a i}^{R}+\frac{\partial}{\partial x^{i}} \xi_{a}, \quad F_{i a}^{L} \rightarrow F_{i a}^{L}-\frac{\partial}{\partial x^{i}} \xi_{a} .
\end{gathered}
$$

In particular, for $\xi$ constant, this is just a constant shift of $E_{a b}$. In general, this transformation of the background fields $E, F^{R}, F^{L}$ shifts the original action (4.1) by the total derivative term

$$
\frac{1}{2 \pi} \int d^{2} z\left(\bar{\partial}\left[\xi_{a}\left(g^{-1} \partial g\right)^{a}\right]-\partial\left[\xi_{a}\left(g^{-1} \bar{\partial} g\right)^{a}\right]\right)
$$

(we use the Bianchi identity $F\left(g^{-1} \partial g, g^{-1} \bar{\partial} g\right)=0$ ).

One of the outstanding problems in nonabelian duality is the nature of the group of duality transformations. As discussed in section 3, since, in general, nonabelian duality transformations take backgrounds with symmetries into background without corresponding symmetries, we do not even know how to perform the inverse transformation. In the case without isotropy, however, it seems that there is a way of finding the inverse using the nonlocal transformations (3.6). One might hope that the transformation (3.6) uniquely defines the coordinates $\lambda$ precisely up to shifts (4.17); as discussed above, these shifts do not change the original ("anti-dual") background.

When the $g$ 's are elements of, e.g., $G \times G \times \ldots \times G=G^{d}$, then we can look for a duality group analogous to $O(d, d)$ when $G$ is $U(1)$; this is currently under investigation. 


\section{An example without isotropy}

The analysis of the previous section did not address the issue of world sheet conformal invariance. A simple example of nonabelian duality without isotropy for conformal field theories arises for the WZNW model on $G \times G$ at level $k$ dualized with respect to a particular nonanomalous $G$ subgroup. Explicitly, we start with

$$
S_{G_{k} \times G_{k}}\left[g_{1}, g_{2}\right]=S_{G_{k}}\left[g_{1}\right]+S_{G_{k}}\left[g_{2}\right],
$$

where $S_{G_{k}}$ is the WZNW action for $G$ at level $k$ :

$$
S_{G_{k}} \equiv \frac{k}{2 \pi}\left[\frac{1}{2} \int d^{2} z \operatorname{tr}\left(g^{-1} \partial g g^{-1} \bar{\partial} g\right)+\Gamma\right],
$$

where $\Gamma$ is the Wess-Zumino term. We gauge the subgroup [11]

$$
g_{1} \rightarrow u^{-1} g_{1}, \quad g_{2} \rightarrow g_{2} u
$$

The gauged action is

$$
\begin{aligned}
S_{\text {gauge }} & =S_{G}\left[f g_{1}\right]+S_{G}\left[g_{2} \bar{f}\right]-S_{G}[f \bar{f}] \\
& =S_{G}\left[g_{1}\right]+S_{G}\left[g_{2}\right]+\frac{k}{2 \pi} \int d^{2} z \operatorname{tr}\left(A \bar{\partial} g_{1} g_{1}{ }^{-1}-g_{2}{ }^{-1} \partial g_{2} \bar{A}+A \bar{A}\right),
\end{aligned}
$$

where $A \equiv f^{-1} \partial f$ and $\bar{A} \equiv-\bar{\partial} \bar{f} \bar{f}^{-1}$, and we have used the PolyakovWiegmann formula 12

$$
S_{G_{k}}[g h]=S_{G_{k}}[g]+S_{G_{k}}[h]+\frac{k}{2 \pi} \int d^{2} z \operatorname{tr}\left(g^{-1} \partial g \bar{\partial} h h^{-1}\right) .
$$

We then add the usual $\lambda F(A, \bar{A})$ term.

With the coordinates $g_{1}, g_{2}$, the action $S_{G \times G}$ (5.1) does not manifestly have the structure of the models discussed in the previous section; to find this structure, we define new variables $g, x$, with $x$ inert under (5.3), as follows:

$$
g \equiv g_{1}, \quad x \equiv g_{2} g_{1}
$$


Then using Polyakov-Wiegmann formula (5.5), the action $S_{G \times G}$ takes the form

$$
S_{G \times G}[g, x]=S_{G}[x]+\frac{k}{2 \pi} \int d^{2} z \operatorname{tr}\left(g^{-1} \partial g g^{-1} \bar{\partial} g-x^{-1} \partial x g^{-1} \bar{\partial} g\right) .
$$

This action has the form (4.1) with $E_{a b}=k \eta_{a b}, F_{i a}^{L}=-k e_{i a}$ where $e$ is the usual left-invariant 1-form of the group, $F_{a i}^{R}=0$, and $F_{i j}(x)$ is the usual background for a $G$-WZNW model. In this form, the gauging (5.4) is given simply by the minimal coupling prescription (4.9), and, after choosing the gauge $g=1$, takes the form (4.10).

For $G=S U(2)$, the resulting dual space is a six-dimensional space with three noncompact coordinates $\lambda$ fibering over $S U(2)$ (coordinatized by $x$ ). Such a background could arise in compactifying strings. It would be interesting to investigate its geometry.

\section{The $G$-dual of the $G$-WZNW model: An example with isotropy}

\subsection{General discussion}

The simplest examples of $G$-WZNW models with isotropy are the duals with respect to the full group $G$. We begin by gauging $S_{G}$ in the standard way [13]:

$$
\begin{aligned}
S_{G}[g] & \rightarrow S_{G / G}[g, f, \bar{f}]=S_{G}[f g \bar{f}]-S_{G}[f \bar{f}] \\
& =S_{G}[g]+\frac{k}{2 \pi} \int d^{2} z \operatorname{tr}\left(A \bar{\partial} g g^{-1}-g^{-1} \partial g \bar{A}+A \bar{A}-A g \bar{A} g^{-1}\right),
\end{aligned}
$$

where $A \equiv f^{-1} \partial f$ and $\bar{A} \equiv-\bar{\partial} \bar{f} \bar{f}^{-1}$, and we have used the PolyakovWiegmann formula (5.5). This action is invariant under

$$
g \rightarrow u^{-1} g u, \quad f \rightarrow f u, \quad \bar{f} \rightarrow u^{-1} \bar{f} .
$$


These transformations do not allow us to reach the gauge $g=1$, and hence the isotropy doesn't vanish. The best we can do is, e.g., to choose $g$ to lie in the Cartan torus.] To find the nonabelian $G$-dual of $G$, we add the standard $\lambda F$ term; further gauge choices to completely fix the gauge invariance can be made on the Lagrange multipliers $\lambda$ themselves [9].

Before gauge fixing, the total action becomes

$$
S=S_{G}[g]+\frac{1}{2 \pi} \int d^{2} z\left(A^{a} \bar{K}_{a}-K_{a} \bar{A}^{a}+A^{a} M_{a b} \bar{A}^{a}\right)
$$

where

$K=k g^{-1} \partial g+\partial \lambda, \quad \bar{K}=k \bar{\partial} g g^{-1}+\bar{\partial} \lambda, \quad M_{a b}=k\left[\eta_{a b}-\operatorname{tr}\left(g^{-1} T_{a} g T_{b}\right)\right]+\lambda_{c} f_{a b}^{c}$,

and indices are raised and lowered by the Killing metric $\eta_{a b}=\operatorname{tr}\left(T_{a} T_{b}\right)$. Integrating out the gauge fields, we find

$$
\tilde{S}[g, \lambda]=S_{G}[g]+\frac{1}{2 \pi} \int d^{2} z\left(K_{a}\left[M^{-1}\right]^{a b} \bar{K}_{b}-\frac{1}{4} \ln (\operatorname{det}[M]) R^{(2)}\right) .
$$

This action is still gauge invariant, and may be gauge fixed by eliminating certain components of $g$ and $\lambda$.

\subsection{The $S U(2)$ example}

For $S U(2)$, we parametrize the group element $g$ as:

$$
g=e^{\frac{i}{2} \theta_{L} \sigma_{3}} e^{\frac{i}{2} \phi \sigma_{2}} e^{\frac{i}{2} \theta_{R} \sigma_{3}}=\left(\begin{array}{cc}
e^{\frac{i}{2}\left(\theta_{L}+\theta_{R}\right)} \cos \frac{\phi}{2} & e^{\frac{i}{2}\left(\theta_{L}-\theta_{R}\right)} \sin \frac{\phi}{2} \\
-e^{-\frac{i}{2}\left(\theta_{L}-\theta_{R}\right)} \sin \frac{\phi}{2} & e^{-\frac{i}{2}\left(\theta_{L}+\theta_{R}\right)} \cos \frac{\phi}{2}
\end{array}\right),
$$

where $\sigma_{a}$ are the Pauli matrices and

$$
\frac{1}{2}\left(\theta_{L} \pm \theta_{R}\right) \in[0,2 \pi), \phi \in[0, \pi)
$$

\footnotetext{
${ }^{7}$ This manifests itself in that the $G / G$ CFT is a nontrivial topological field theory.
} 
are Euler angles. We normalize the generators as $T_{a} \equiv \frac{i}{2} \sigma_{a}$. Under an infinitesimal transformation $\delta g=[g, \epsilon], \epsilon \equiv \epsilon^{a} T_{a}$, the Euler angles transform as:

$$
\begin{aligned}
\delta \phi & =-\epsilon_{1}\left(\sin \theta_{L}+\sin \theta_{R}\right)+\epsilon_{2}\left(\cos \theta_{L}-\cos \theta_{R}\right) \\
\delta \theta_{R} & =\epsilon_{3}+\frac{\epsilon_{1}}{\sin \phi}\left(\cos \theta_{L}-\cos \phi \cos \theta_{R}\right)+\frac{\epsilon_{2}}{\sin \phi}\left(\sin \theta_{L}+\cos \phi \sin \theta_{R}\right) \\
\delta \theta_{L} & =-\epsilon_{3}+\frac{\epsilon_{1}}{\sin \phi}\left(\cos \theta_{R}-\cos \phi \cos \theta_{L}\right)-\frac{\epsilon_{2}}{\sin \phi}\left(\sin \theta_{R}+\cos \phi \sin \theta_{L}\right) .
\end{aligned}
$$

Note that, e.g., $\epsilon_{1}$ and $\epsilon_{3}$ can be used to go to a gauge $\theta_{R}=\theta_{L}=0$, but that in that gauge, $\epsilon_{2}$ does not act on any of the Euler angles. This is an explicit demonstration of the nontrivial isotropy of this gauging.

We now turn to the Lagrange multiplier $\lambda \equiv \lambda^{a} T_{a}$; it transforms as $\delta \lambda=$ $[\lambda, \epsilon]$, or, explicitly

$$
\delta \lambda_{1}=\lambda_{2} \epsilon_{3}-\lambda_{3} \epsilon_{2}, \quad \delta \lambda_{2}=\lambda_{3} \epsilon_{1}-\lambda_{1} \epsilon_{3}, \quad \delta \lambda_{3}=\lambda_{1} \epsilon_{2}-\lambda_{2} \epsilon_{1} .
$$

We can fix the $\epsilon_{2}$ transformation by choosing, e.g., a gauge $\lambda_{3}=0$.

Now, evaluating the dual action (6.5) for $S U(2)$ with the particular gauge choice $\theta_{R}=\theta_{L}=\lambda_{3}=0$, we find a $\sigma$-model with no antisymmetric tensor and with a metric

$d s^{2}=\frac{k}{4}\left((d \phi)^{2}+\frac{1}{\lambda_{1}^{2} \sin ^{2} \frac{\phi}{2}}\left[4 \sin ^{4} \frac{\phi}{2}\left(d \lambda_{2}\right)^{2}+\left(\frac{1}{k} \lambda_{1} d \lambda_{1}+\left[\frac{1}{k} \lambda_{2}+\phi-\sin \phi\right] d \lambda_{2}\right)^{2}\right]\right)$.

We also find a dilaton

$$
\Phi=\ln \left(\lambda_{1}^{2} \sin ^{2} \frac{\phi}{2}\right) .
$$

We have checked explicitly that this background solves the one-loop $\beta$-function equations (for a review, see [14):

$$
R_{a b}=\nabla_{a} \nabla_{b} \Phi
$$

The singularities at $\lambda_{1}=0$ and at $\phi=0$ clearly arise because our gauge choice breaks down at these points, and hence are an artifact of the $\sigma$-model description of the CFT. 


\section{Comments and Discussion}

In this paper, we have discussed aspects of nonabelian duality. We found that it is not an exact symmetry of the conformal field theory, and conjectured that it is a map between different conformal field theories. We also found that, though in general nonabelian duality does not preserve the isometries of the original target space, the dual theory has a nonlocal symmetry that somehow encodes the information about its more symmetric progenitor. We emphasized the key role of isotropy, and found that most WZNW models have vanishing isotropy at generic points. For such models, we sketched how the nonlocal symmetry could be used to find the inverse nonabelian dual transformation. We were not able to solve the outstanding problem of finding the group structure of nonabelian duality transformations. We also worked out several examples in some detail, both with and without isotropy.

\section{Note Added}

We thank the referee for suggesting that we expand the discussion of whether the dual of a conformal field theory is conformal. We argue that this is the case as long as the gauging of the isometry does not introduce any anomalies that cannot be cancelled by modified transformations of the Lagrangemultiplier fields $\lambda$. Our argument runs as follows: whether a theory is conformally invariant has to do with the short distance behavior of the theory. In particular, if it is conformal on a topologically trivial world sheet (modulo possible global anomalies), it is conformal; however, on a topologically trivial world sheet, the nonabelian dual of a theory is the same as the original theory; non-trivial flat connections arise only on world sheets that are not simply connected. Hence, if the gauging procedure itself does not break conformal invariance, the nonabelian dual of a conformal field theory must be conformal. In this work we described examples that fall into this category, namely, (non-anomalous) gauged WZNW models.

After the completion of our original manuscript, [15] appeared. It includes an example of a CFT whose nonabelian dual is not conformal; however, the 
group gauged in that example has structure constants that are not traceless

$\left(f_{a b}^{a} \neq 0\right)$; this gives rise to an anomaly (not the standard one), and hence the argument given above does not apply.

\section{Acknowledgements}

We would like to thank Warren Siegel for discussions, and the Institute for Theoretical Physics at Santa Barbara, where part of this work was done, for its warm hospitality. AG would like to thank Elias Kiritsis and Gabriele Veneziano for discussions, and the Institute for Theoretical Physics at Stony Brook, where part of this work was done, for its warm hospitality. This work was supported in part by NSF grant No. PHY 9211367. 


\section{References}

[1] A. Giveon, E. Rabinovici and G. Veneziano, Nucl. Phys. B322 (1989) 167 ;

A. Shapere and F. Wilzcek, Nucl. Phys. B320 (1989) 669;

A. Giveon, N. Malkin and E. Rabinovici, Phys. Lett. 220B (1989) 551.

[2] S. Ferrara, D. Lüst, A. Shapere and S. Theisen, Phys. Lett. B233 (1989) 147;

J. Lauer, J. Maas and H.P. Nilles, Phys. Lett. B226 (1989) 251; Nucl. Phys. B351 (1991) 353;

W. Lerche, D. Lüst and N.P. Warner, Phys. Lett. B231 (1989) 418;

S. Ferrara, D. Lüst and S. Theisen, Phys. Lett. B233 (1989) 147;

A. Giveon, N. Malkin and E. Rabinovici, Phys. Lett. B238 (1990) 57;

A. Font, L. Ibanez, D. Lust and F. Quevedo, Phys. Lett. B245 (1990) 401;

A. Giveon and D.-J. Smit, Nucl. Phys. B349 (1991) 168;

A. Giveon and M. Porrati, Phys. Lett. B246 (1990) 54; Nucl. Phys. B355 (1991) 422;

P. Candelas X.C. de la Ossa, P.S. Green and L. Parkes, Nucl. Phys. B359 (1991) 21;

T. Kugo and B. Zwiebach, Prog. Theor. Phys. 87 (1992) 801.

[3] A. Giveon and M. Roček, Nucl. Phys. B380 (1992) 128.

[4] T. Buscher, Phys. Lett. 159B (1985) 127; Phys. Lett. 194B (1987) 59; Phys. Lett. 201B (1988) 466.

[5] M. Roček and E. Verlinde, Nucl. Phys. B373 (1992) 630.

[6] T. Buscher, Ph.D. Thesis, May 1988, unpublished.

[7] B.E. Fridling and A. Jevicki, Phys. Lett. B134 (1984) 70.

[8] E.S. Fradkin and A.A. Tseytlin, Ann. Phys. 162 (1985) 31. 
[9] X.C. de la Ossa and F. Quevedo, Nucl. Phys. B403 (1993) 377.

[10] E. Cremmer, J. Scherk, and S. Ferrara, Phys. Lett. B74 (1978) 256;

E. Cremmer and B. Julia, Nucl. Phys. B159 (1979) 141;

E. Cremmer, J. Scherk, and J.H. Schwarz, Phys. Lett. B84 (1979) 83.

[11] E. Witten, Commun. Math. Phys. 144 (1992) 189.

[12] A.M. Polyakov and P.B. Wiegmann, Phys. Lett. B131 (1984) 121; B141 (1984) 223.

[13] K. Bardakci, E. Rabinovici, and B. Säring, Nucl. Phys. B299 (1988) 151;

K. Gawedski and A. Kupianen, Nucl. Phys. B320 (1989) 625.

[14] M.B. Green, J.H. Schwarz and E. Witten, 'Superstring theory' (Cambridge Univ. Press, Cambridge, 1987).

[15] M. Gasperini, R. Ricci and G. Veneziano, A problem with nonabelian duality?, preprint CERN-TH-6960-93, hep-th/9308112. 